\title{
The transition from order to disorder in Voronoi Diagrams with applications
}

\author{
L. Zaninetti ${ }^{1}$
}

${ }^{1}$ Department of Physics, via P.Giuria 1, I-10125 Turin,Italy

Correspondence: L. Zaninetti, Department of Physics, via P.Giuria 1, I-10125 Turin,Italy. E-mail: zaninetti@ph.unito.it

Received: August 21, 2018

Accepted: September 26, 2018

Online Published: November 30, 2018

doi:10.5539/apr.v10n6p82

URL: https://doi.org/10.5539/apr.v10n6p82

\begin{abstract}
The transition from ordered to disordered structures in Voronoi tessellation is obtained by perturbing the seeds that were originally identified with two types of lattice in $2 \mathrm{D}$ and one type in $3 \mathrm{D}$. The area in $2 \mathrm{D}$ and the volume in $3 \mathrm{D}$ are modeled with the Kiang function. A new relationship that models the scaling of the Kiang function with a geometrical parameter is introduced. A first application models the local structure of sub- and supercritical ammonia as function of the temperature and a second application models the volumes of cosmic voids.
\end{abstract}

Keywords: Voronoi diagrams, Monte Carlo methods, Cell-size distribution

\section{Introduction}

The seeds in 1D, 2D and 3D Voronoi Diagrams are usually taken to be randomly distributed, the so called Poisson-Voronoi tessellations (PVT) (Okabe et al., 2000). A first generalization of the PVT are the quasi random seeds, such as the Sobol seeds (Sobol, 1967; Bratley \& Fox, 1988; Zaninetti, 1992, 2009; Zaninetti \& Ferraro, 2015) and the eigenvalues of complex random matrices (Le Caer \& Ho, 1990). A second major generalisation modifies regular structures to produce non-Poissonian seeds for the Voronoi tessellation (NPVT). We select some methods, including perturbation of cubic structures (Lucarini), generation of seeds with controlled regularity (Zhu et al., 2014), an information geometric model to simulate graphene (Dodson, 2015), a 3D topological analysis (Lazar, Han, \& Srolovitz, 2015) and two-dimensional perturbed systems (Leipold et al., 2016).

The rest of this paper is organised as follows: In section 2, we first review the existing formulae which model the areas and volumes in 2D/3D PVT. Afterwards, we introduce two models for NPVT, see Section 3. Two applications are discussed in Section 4.

\section{Useful formulae in PVT}

The probability density function (PDF) for segments (1D) in PVT is modeled by a gamma variate

$$
H(x ; c)=\frac{c}{\Gamma(c)}(c x)^{c-1} \exp (-c x),
$$

where $0 \leq x<\infty, c>0$ and $\Gamma(c)$ is the gamma function with argument $\mathrm{c}$, see formula (5) in Kiang (1966). In the case of $1 \mathrm{D}, \mathrm{c}=2$ which is an analytical result. Conversely the PDF for areas in $2 \mathrm{D}$ and volumes in $3 \mathrm{D}$ was conjectured to follow the above gamma variate with $c=4$ and $c=6$. Later on, the "Kiang conjecture" was refined by Ferenc and Nda (2007) with the following PDF

$$
F N(x ; d)=\text { Const } \times x^{\frac{3 d-1}{2}} \exp (-(3 d+1) x / 2),
$$

where

$$
\text { Const }=\frac{\sqrt{2} \sqrt{3 d+1}}{22^{3 / 2 d}(3 d+1)^{-3 / 2 d} \Gamma(3 / 2 d+1 / 2)},
$$

and $d(d=1,2,3)$ represents the dimension of the considered space. This PDF allows us to fix the "Kiang conjecture" in $\mathrm{c}=3.5$ and $\mathrm{c}=5$ for the $2 \mathrm{D}$ and $3 \mathrm{D}$ PVT case. A typical 2D result is reported in Figure 1 for the reduced area distribution when the Poissonian seeds are 20000, $d=2$ and $c=3.50$.

The theoretical expected number of edges of a typical 2D cell is six, see Table 5.5.1 in Okabe et al. (2000) and our results are reported in Figure 2, in which the averaged edges are 6.125. 


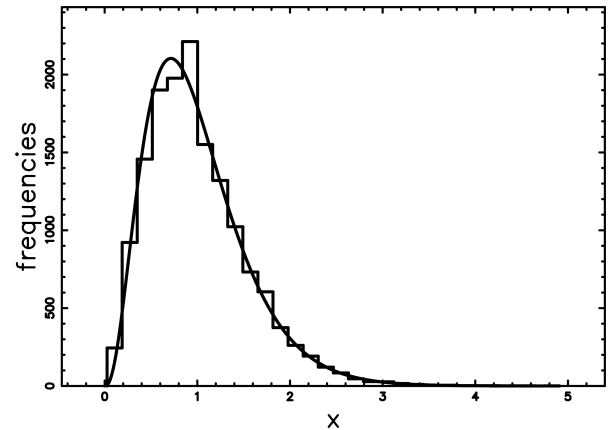

Figure 1: Histogram (step-diagram) of the 2D PVT reduced area distribution.

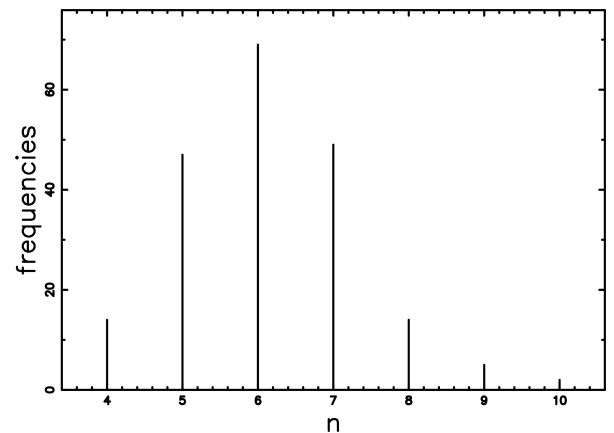

Figure 2: Histogram of the expected number of edges in the 2D PVT.

In the 3D case Figure 3 reports the histogram for the volume distribution when the Poissonian seeds are $4096, d=3.03$ and $c=5.05$. and Figure 4 the histogram of the number of faces with average value 14.897. A comparison should be done with the expected value 15.535, see Table 5.5.2 in Okabe et al. (2000).

\subsection{The lognormal distribution}

The lognormal PDF, $f_{L N}$, is

$$
f_{L N}(x ; m, \sigma)=\frac{\mathrm{e}^{-\frac{1}{2 \sigma^{2}}\left(\ln \left(\frac{x}{m}\right)\right)^{2}}}{x \sigma \sqrt{2 \pi}},
$$

where $m$ is the median and $\sigma$ the shape parameter, see Evans, Hastings, and Peacock (2000). The distribution function $(\mathrm{DF}), F_{L N}$, is

$$
F_{L N}(x ; m, \sigma)=\frac{1}{2}+\frac{1}{2} \operatorname{erf}\left(\frac{1}{2} \frac{\sqrt{2}(-\ln (m)+\ln (x))}{\sigma}\right),
$$

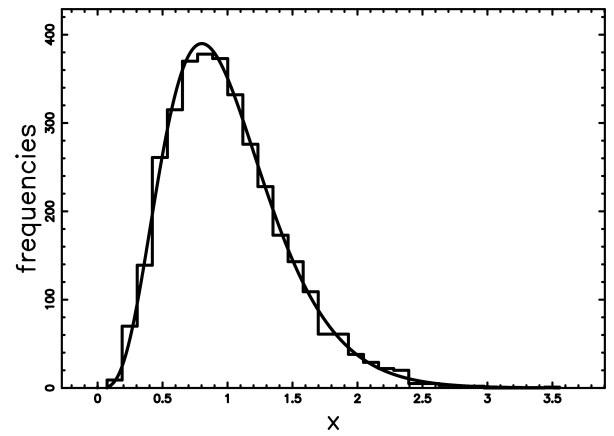

Figure 3: Histogram (step-diagram) of the 3D PVT reduced volume distribution. 


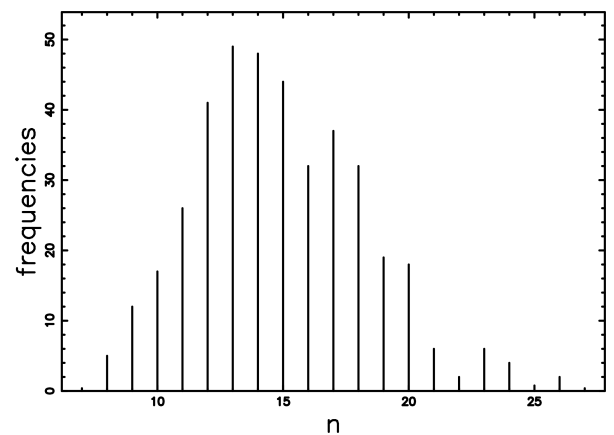

Figure 4: Histogram of the expected number of faces in the 3D PVT.

where $\operatorname{erf}(\mathrm{x})$ is the error function, defined as

$$
\operatorname{erf}(x)=\frac{2}{\sqrt{\pi}} \int_{0}^{x} e^{-t^{2}} d t
$$

see Abramowitz \& Stegun (1965).

\section{Non-Poissonian case}

Two regular geometrical models are perturbed to have NPVT and a function which models the transition from order to disorder is introduced.

\subsection{The modified lattice case}

To have more flexible seeds we introduce the adjustable non Poissonian seeds (LNPVT), which can be computed both in 2D and 3D following an algorithm introduced in Zaninetti and Ferraro (2015). The algorithm is now outlined:

1. The process starts by inserting the seeds on a 2D/3D regular Cartesian grid with equal distance $\delta$ between one point and the following one.

2. A random radius is generated according to the half Gaussian,$H N(x)$, which is defined in the interval $[0, \infty]$

$$
H N(x ; s)=\frac{2}{s(2 \pi)^{1 / 2}} \exp \left(-\frac{x^{2}}{2 s^{2}}\right) \quad 0<x<\infty .
$$

A random direction is chosen in 2D/3D and the two/three Cartesian coordinates of the generated radius are evaluated. These two/three small Cartesian components are added to the regular 2D/3D grid which represent the seeds. To have small corrections, we express $s$ in $\delta$ units.

3. We now have $N$ seeds and we can eliminate a given number of seeds, $N_{\text {hole }}$, according to the rule $N_{\text {hole }}=N \times p_{\text {hole }}$. This elimination of seeds will allow a more disordered distribution of areas/volumes; otherwise specified $p_{\text {hole }}=0$.

Figure 5 reports an example of 2D tessellation from LNPVT in which $s=0.1$ and we have 400 seeds.

An example of the presence of holes in this network for the seeds is reported in Figure 6 where original seeds are 400 , $s=0.1$ and $p_{\text {hole }}=0.19$.

Another case uses the lattice points shifted of delta/2 between one row and the following row as a first point of the suggested algorithm, we define it as triangular non Poissonian tessellation, TNPVT. This tessellation will be produced in 2D irregular hexagons, see Figure 7 where we have 400 seeds and $s=0.1$.

\subsection{Order-disorder transition}

We can model the order disorder transition introducing the following function for $c$ of Kiang as in equation (1)

$$
c\left(s ; c_{\min }, c_{\max }, a\right)=c_{\max }-\left(c_{\max }-c_{\min }\right)\left(1-\mathrm{e}^{-a s}\right),
$$

where $s$ is the scale of the half Gaussian, see equation (7), $c_{\max }$ and $c_{\min }$ are the maximum and minimum value for $c$ of Kiang and $a$ is a scale to be found from the fitting procedure. A typical 2D example for $c$ as function of $s$ is reported in 


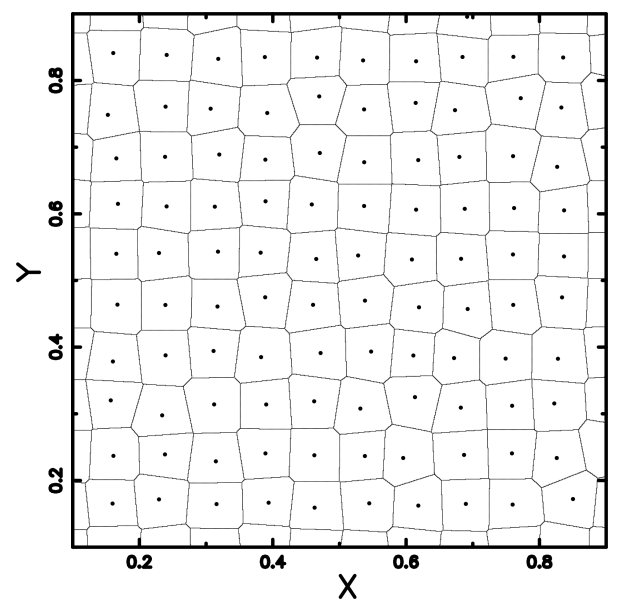

Figure 5: An example of 2D LNPVT.

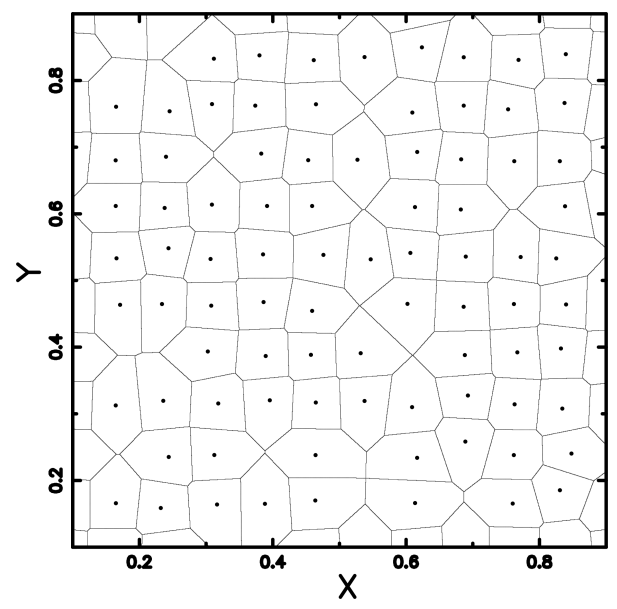

Figure 6: An example of 2D LNPVT.

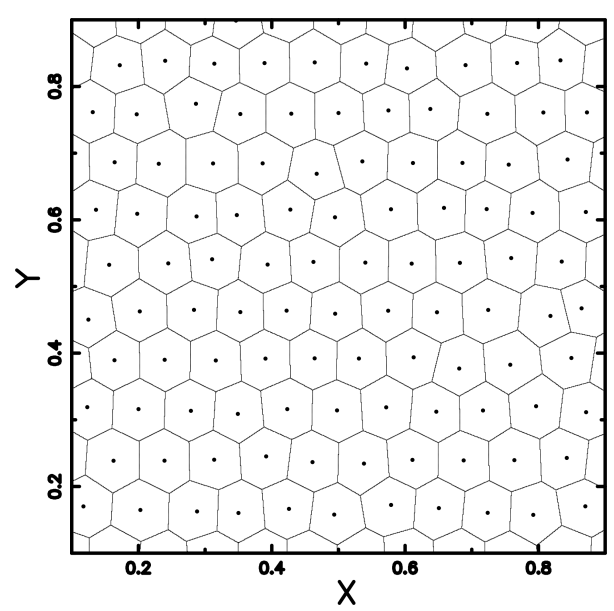

Figure 7: An example of 2D TNPVT. 


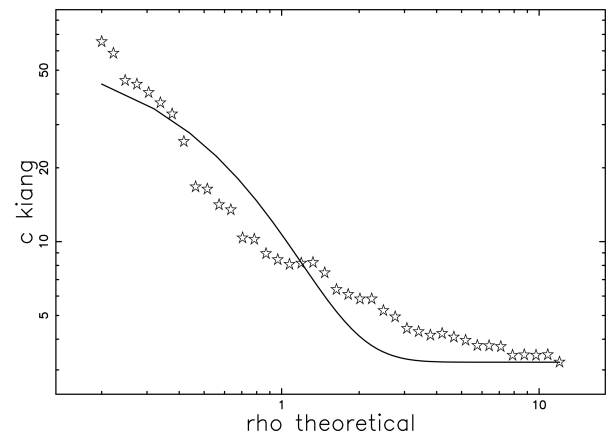

Figure 8: The Kiang parameter $c$ as function of $s$ for 2D LNPVT.

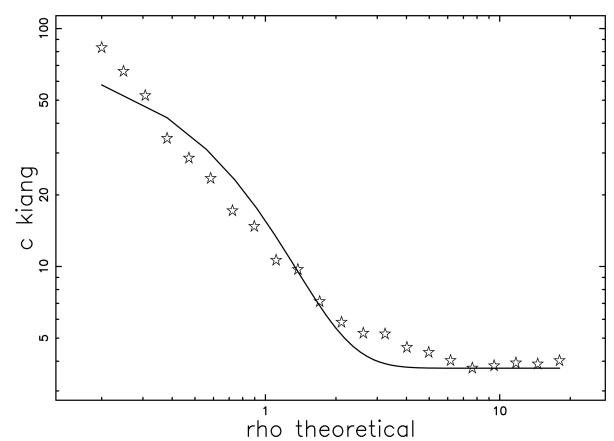

Figure 9: The Kiang parameter $c$ as function of $s$ for 2D TNPVT.

Figure 8 for the LNPVT case where $c_{\min }=3.22$ (the minimum), $c_{\max }=65.33$ (the maximum) and $a=2.11$ and in Figure 9 for the LNPVT case when $c_{\min }=3.73, c_{\max }=83.06$ and $a=1.9$.

A 3D example for $c$ as function of $s$ is reported in Figure 10 for the LNPVT case when $c_{\min }=3.34, c_{\max }=454.12$ and $a=4.25$.

\section{Applications}

Two applications are presented.

4.1 An application to chemistry

The local structure of sub- and supercritical ammonia with $250 \mathrm{~K}<T<500 \mathrm{~K}$ has been extensively analyzed by Idrissi et al. (2011) and Figure 11 reports the $c$ of Kiang as function of the temperature for Figure 5 in Idrissi et al. (2011). These values for $c$ of Kiang are obtained from Figure 5 in Idrissi et al. (2011) and we report as an example the deduction of the first couple: $\sigma_{V}\left(\AA^{3}\right)=4.33, T(K)=250.9, \sigma=\frac{4.33}{250.9}=0.017$ which means $c=3356$. Figure 12 reports both the chemical and theoretical data: data with stars as deduced from Figure 5 in Idrissi et al. (2011) and full line derived

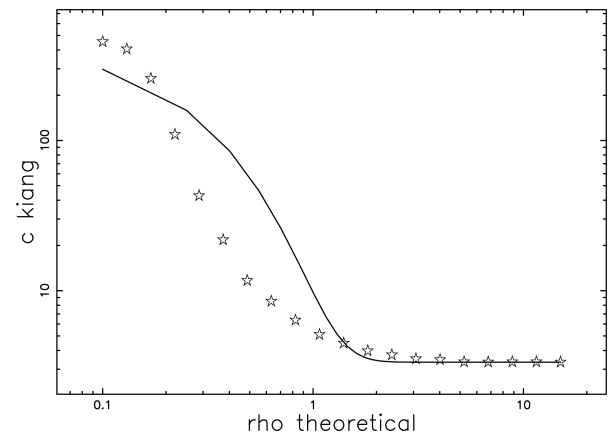

Figure 10: The Kiang parameter $c$ as function of $s$ for 3D LNPVT. 


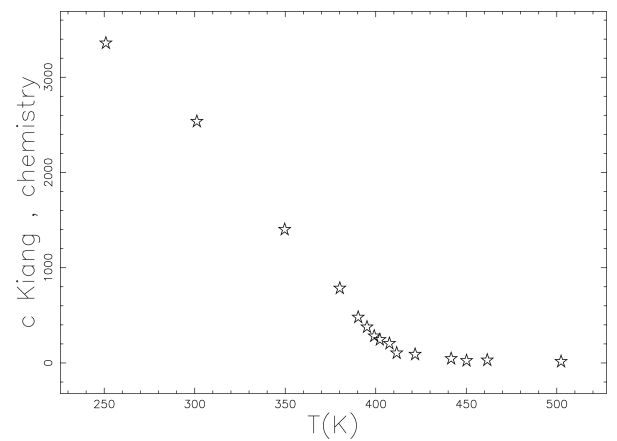

Figure 11: The Kiang parameter $c$ as function of $\mathrm{T}$.

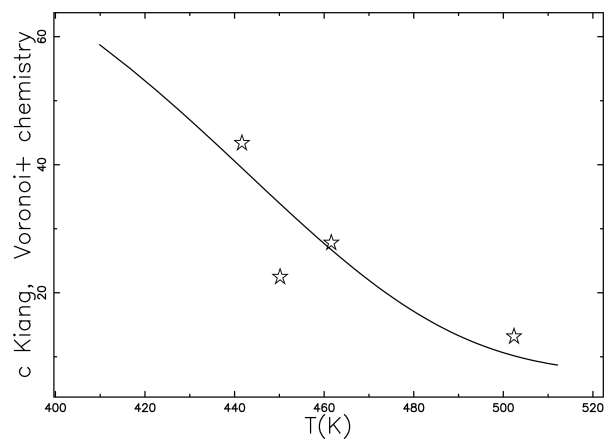

Figure 12: The Kiang parameter $c$ as function of T.

coupling equations (8) and (9).

To set up the theoretical data from equation (8) the following relationship has been used

$$
T=512 \times s^{0.096} K \text {. }
$$

\subsection{An application to cosmic voids}

The catalog of the Baryon Oscillation Spectroscopic Survey (BOSS), see Mao et al. (2017), reports the volume in units of $M p c^{3} / h$ of 1228 cosmic voids where $h=H_{0} / 100$ and the Hubble constant, $H_{0}$, is expressed in 0units. The numerical analysis gives $c=0.02$ for the distribution of the reduced volume of cosmic voids and Figure 13 reports the distribution function (DF) of the lognormal, see equation (5) with parameters as in Table 1.

These results for the cosmic voids can be simulated analyzing the distribution of the reduced volumes in 3D tessellation from LNPVT, see Figure 14, where we have 195112 original seeds, $s=10$ and $p_{\text {hole }}=0.861$. In this figure, the lognormal DF is the full line and the parameters are as in Table 1 , where $D$ is the maximum distance between theoretical and observed

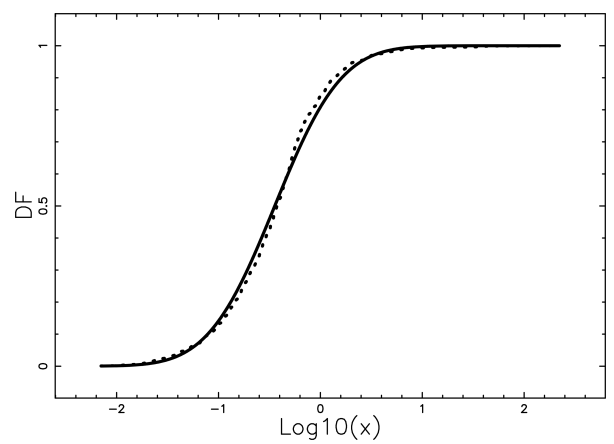

Figure 13: Empirical DF of reduced voids distribution for BOSS (dotted line) and lognormal DF (full line). 


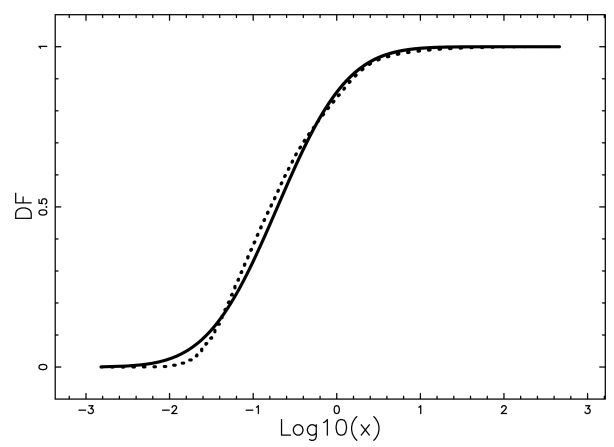

Figure 14: Empirical DF of reduced volume distribution for 3D LNPVT.

$D F$, and $P_{K S}$, is the significance level, in the K-S test.

Table 1. Lognormal parameters for reduced volumes of cosmic voids and relative LNPVT simulation.

\begin{tabular}{cccc}
\hline case & parameters & $\mathrm{D}$ & $P_{K S}$ \\
\hline astronomical observations & $m=0.356 ; \sigma=1.182$ & 0.049 & 0.005 \\
LNPVT simulation & $m=0.195 ; \sigma=1.523$ & 0.055 & 0.003 \\
\hline
\end{tabular}

\section{Conclusions}

We perturbed the 2D seeds identified by a Cartesian and a triangular lattice, as well the 3D seeds of a Cartesian lattice. The probability to have some holes in the resulting 2D/3D seeds is introduced. The area and volume distribution are modeled by the one parameter Kiang's PDF. The transition from order to disorder is parametrised by a geometrical variable, which regulates the strength of the perturbation of the ordered seeds. Two applications are presented: one to the local structure of sub- and supercritical ammonia and the second one to the volumes of cosmic voids.

\section{References}

Abramowitz, M., \& Stegun, I. A. (1965). Handbook of Mathematical Functions with Formulas, Graphs, and Mathematical Tables. New York: Dover.

Bratley, P., \& Fox, B. L. (1988). Implementing Sobols quasirandom sequence generator. ACM Trans. Math. Softw., 14(1), $88 \mathrm{C} 100$.

Dodson, C. T. J. (2015). A Model for Gaussian Perturbations of Graphene. Journal of Statistical Physics, 161,933 C941.

Evans, M., Hastings, N., \& Peacock, B. (2000). Statistical Distributions - third edition. John Wiley \& Sons Inc, New York.

Ferenc, J. S., \& Nda, Z. (2007). On the size distribution of Poisson Voronoi cells. Physica A: Statistical Mechanics and its Applications, 385(2), 518-526.

Idrissi, A., Vyalov, I., Kiselev, M., Fedorov, M. V., \& Jedlovszky, P. (2011). Heterogeneity of the local structure in sub-and supercritical ammonia: A voronoi polyhedra analysis. The Journal of Physical Chemistry B, 115(31), 9646C9652.

Kiang, T. (1966). Random fragmentation in two and three dimensions. Zeitschrift fur Astrophysik, 64, 433.

Lazar, E. A., Han, J., \& Srolovitz, D. J. (2015). Topological framework for local structure analysis in condensed matter. Proceedings of the National Academy of Science, 112, E5769CE5776.

Le Caer, G., \& Ho, J. S. (1990). The voronoi tessellation generated from eigenvalues of complex random matrices. Journal of Physics A: Mathematical and General, 23(14), 3279.

Leipold, H., Lazar, E. A., Brakke, K. A., \& Srolovitz, D. J. (2016). Statistical topology of perturbed two-dimensional lattices. Journal of Statistical Mechanics: Theory and Experiment, 4, 043103.

Lucarini, V. (2009). Three-dimensional random voronoi tessellations: From cubic crystal lattices to poisson point processes. Journal of Statistical Physics, 134(1), 185. 
Mao, Q., Berlind, A. A., Scherrer, R. J., Neyrinck, M. C., Scoccimarro, R., Tinker, J. L., ... \& Malanushenko, E. (2017). A cosmic void catalog of SDSS DR12 BOSS galaxies. The Astrophysical Journal, 835(2), 161.

Okabe, A., Boots, B., Sugihara, K., \& Chiu, S. (2000). Spatial tessellations. Concepts and Applications of Voronoi diagrams (2nd ed.). Wiley, Chichester, New York.

Sobol, I. M. (1967). The distribution of points in a cube and the approximate evaluation of integrals. USSR Comp. Math. Math. Phys., 7(4), 86C112.

Zaninetti, L. (1992). The Voronoi tessellation generated from different distributions of seeds. Physics Letters A, 165(2), 143-147.

Zaninetti, L. (2009). Poissonian and non-Poissonian Voronoi diagrams with application to the aggregation of molecules. Physics Letters A, 373(36), 3223-3229.

Zaninetti, L., \& Ferraro, M. (2015). On Non-Poissonian Voronoi Tessellations. Applied Physics Research, 7, $108 \mathrm{C} 124$.

Zhu, H. X., Zhang, P., Balint, D., Thorpe, S. M., Elliott, J. A., Windle, A. H., \& Lin, J. (2014). The effects of regularity on the geometrical properties of Voronoi tessellations. Physica A Statistical Mechanics and its Applications, 406, $42 \mathrm{C} 58$.

\section{Copyrights}

Copyright for this article is retained by the author(s), with first publication rights granted to the journal.

This is an open-access article distributed under the terms and conditions of the Creative Commons Attribution license (http://creativecommons.org/licenses/by/4.0/). 